\title{
Grade 2 and 3 Obesity and Diagnosed Prostate Cancer in Middle-Aged and Elderly Men: An Epidemiologic Study with Stratified Multistage Sampling Design
}

\author{
Xuefeng Liu, ${ }^{1,2}$ Amal Khoury, ${ }^{3}$ Joshua Longcoy, ${ }^{1}$ and Joseph Ikekwere ${ }^{1}$ \\ ${ }^{1}$ Department of Biostatistics and Epidemiology, East Tennessee State University, 149 H Lamb Hall, Johnson City, TN 37614, USA \\ ${ }^{2}$ Institute for Quantitative Biology, East Tennessee State University, Johnson City, TN 37614, USA \\ ${ }^{3}$ Department of Health Services, Policy and Management, East Tennessee State University, Johnson City, TN 37614, USA
}

Correspondence should be addressed to Xuefeng Liu; lix01@etsu.edu

Received 28 October 2013; Accepted 8 December 2013; Published 8 January 2014

Academic Editors: V. Bencko, A. Finckh, and R. Zhao

Copyright (C) 2014 Xuefeng Liu et al. This is an open access article distributed under the Creative Commons Attribution License, which permits unrestricted use, distribution, and reproduction in any medium, provided the original work is properly cited.

\begin{abstract}
Background. The association of obesity with the odds of diagnosed prostate cancer (DPC) is inconclusive. Whether grade 2 or grade 3 obesity is associated with increased odds of DPC has not been investigated. Design and Methods. Cross-sectional data of 7,974 subjects aged $\geq 40$ years were collected from the National Health and Nutrition Examination Survey 2001-2010. Odds ratios (ORs) of DPC associated with grade 2 or grade 3 obesity were estimated by conducting weighted logistic regression models. Results. The unadjusted rates of DPC did not differ significantly over grades of BMI $(P=0.7044)$. After adjustment for different groups of potential confounding factors, grade 2 or grade 3 obesity was not significantly associated with the odds of DPC with ORs changing from 0.62 to 0.69 for grade 2 obesity and from 0.81 to 1.09 for grade 3 obesity. Moreover, morbid obesity (grade 2 and 3 obesity combined) was not linked to the odds of DPC. Conclusion. Grade 2 or grade 3 obesity was not associated with the odds of DPC. Whether they are associated with a substantially increased risk of high-grade DPC needs to be further investigated as accumulating evidence has shown that obesity increases the risk of high-grade disease.
\end{abstract}

\section{Introduction}

Prostate cancer is the most commonly diagnosed non-skin cancer and the second leading cause of cancer death in American men $[1,2]$. It is estimated that about one man in 6 will get prostate cancer and one man in 36 will die of this disease in the United States (US) [3]. Despite high comorbidities associated with prostate cancer, most men diagnosed with prostate cancer have survived from it. This should benefit from more cases being diagnosed in the early state due to the development of the prostate specific antigen (PSA) testing. While we do not know exactly what causes prostate cancer, we do know that certain risk factors are linked to the disease. Identification of risk factors of prostate cancer would be crucial for prevention and control of the disease. The established risk factors for prostate cancer include age, race/ethnicity, family history, and westernization [4-6]. Other potential factors need to be further investigated.
The high prevalence of obesity is becoming a pandemic problem in the US. Medical costs associated with obesity were estimated at $\$ 147$ billion, and medical costs for obese people were $\$ 1,429$ higher than those of normal weight [7]. Obesity may increase the risk of a number of health conditions including hypertension, adverse lipid concentrations, heart disease, stroke, type 2 diabetes, and certain types of cancer [8]. Although weight loss reduces the risk and recurrence of breast cancer particularly in postmenopausal women, evidence for the association between obesity and prostate cancer risk in men is still inconclusive. Some studies have reported the inverse association of obesity with the risk of prostate cancer [9-11], and some indicate no significant association [12-14]. Other studies associate obesity with the increased risk of prostate cancer, particularly high-grade cancer [11, 15]. Inconsistent findings from previous studies warrant further research of the relation between obesity and prostate cancer in a nationally representative sample. Moreover, although 
many prospective and case-control studies have examined the role of obesity in prostate cancer etiology, little information is available on whether morbid obesity (grade 2 and 3 obesity) is associated with prostate cancer, particularly in middle-aged and elderly men at higher risk of prostate cancer.

In this study, we collected data on body weight, height, and diagnosed prostate cancer from the National Health and Examination Survey (NHANES) 2001-2010. The associations of grade 2 and 3 obesity with the odds of diagnosed prostate cancer (DPC) were examined in men aged $\geq 40$ years. We hypothesized that morbid obesity was associated with increased odds of DPC in middle-aged and elderly men. As the population is aging and prostate cancer is more prevalent in men aged $\geq 40$ years, a link between morbid obesity and prostate cancer risk may have clinic implications in understanding prostate cancer genesis and screening strategies and reducing the mortality related to prostate cancer in the high-risk population.

\section{Methods}

2.1. Study Design. The continuous NHANES program has been conducted by the National Center for Health Statistics and Centers for Disease Control and Prevention (CDC) since 1999. It includes a continuous series of two-year cross-sectional, nationally representative health examination surveys. Each survey used a complex, stratified, multistage probability cluster sampling design to obtain a nationally representative sample of the US civilian noninstitutionalized population [16]. The NHANES sampling procedure consisted of 4 stages, including selections of primary sampling units (single counties or groups of contiguous counties), segments within selected counties (generally city blocks or their equivalents), random sample of households within segments, and random sample of individuals residing in selected households [17]. Approximately 10,000 persons from 30 counties across the country were examined in each cycle of survey. The survey included two sections: interview and examination. Interview was performed in the participants' home to obtain information regarding sociodemographic characteristics and history of diseases. Examination was conducted in the mobile examination center to obtain examination and clinical/laboratory measurements.

2.2. Study Participants. Since individuals selected in 19992001 did not participate in the PSA testing for prostate cancer screening, the participants in this cycle were not considered in the present study. We included 5 cycles of NHANES data from interviews and examinations collected during 2001-2010. In NHANES 2001-2010, overall response rates of participants for interview and examination were $83.4 \%$ and $78.6 \%$, respectively. The combined survey sample consisted of 8,457 men aged $\geq 40$ years, of whom all were both interviewed and examined. After removing 483 participants without information for prostate cancer screening, we had 7,974 (377 cases) middle-aged and elderly men included in the study. All participants provided informed consent, and the data received approval from the CDC Institutional
Review Board and Research Ethics Review Board to ensure confidentiality.

2.3. Outcome Measure: Diagnosed Prostate Cancer. Diagnosed prostate cancer (DPC) was defined by a positive response to any of these questions, "Have you ever been told by a doctor or health professional that you have prostate cancer?" "Have you had surgery for prostate cancer?" "Have you ever had radiation treatments for prostate cancer?" or "Have you ever been prescribed medicines for prostate cancer?" A participant had DPC if she/he answered one of the above questions by "Yes." This definition of DPC did not include patients with undiagnosed prostate cancer.

2.4. Primary Factors: Grade 2 and 3 Obesity. Weight and height were measured in a mobile examination center using standardized techniques and equipment. Body mass index (BMI) was calculated as weight in kilograms divided by height in meters squared, rounded to the nearest tenth. For men aged 40 years or older, overweight was defined as a BMI of 25.0 to $29.9 \mathrm{~kg} / \mathrm{m}^{2}$ and obesity was defined as a BMI of $30.0 \mathrm{~kg} / \mathrm{m}^{2}$ or higher. Obesity may be divided into grade 1 , grade 2 , and grade 3 . A man was determined to have grade 2 obesity if his BMI was from 35.0 to $39.9 \mathrm{~kg} / \mathrm{m}^{2}$, and grade 3 obesity if his BMI was from $40.0 \mathrm{~kg} / \mathrm{m}^{2}$ or higher [18].

2.5. Other Factors. Individuals were grouped by age at the interview: middle-aged men (40-59 years) and elderly men (60 years or older). Race and ethnicity were self-reported; for the purposes of this study, race and ethnicity were classified as non-Hispanic white, non-Hispanic black, Hispanic (including Mexican Americans and other Hispanics), and other. The participant's level of education was categorized as receiving education of high school or less and college or above, based on the number of years in school. Persons were defined to be poor if the family's poverty index ratio $(\mathrm{PIR})<1.0$; the PIR was the ratio of the family's total income to the family's appropriate poverty threshold. Alcohol use was categorized as "Yes" (current drinking) and "No" (not drinking). History of smoking was obtained from household interview for the studied men. Smokers were defined as subjects who smoked $\geq 100$ cigarettes in their lives. Current smokers included smokers who reported current smoking; former smokers included smokers who did not report current smoking.

2.6. Statistical Analyses. Since NHANES included a series of surveys using stratified multistage clustered sampling design, sampling weights were incorporated into data analyses to generalize the results to the represented population in the US. Calculation of sampling weights took into account unequal probabilities of selection resulting from the sample design, nonresponse, and noncoverage. All analyses considered the complex sampling design and differential sampling probabilities.

The rates and $95 \%$ confidence intervals (CI) of obesity and DPC by individual characteristics were estimated by integrating sampling weights and complex sampling design. 
TABLE 1: Unadjusted rates and 95\% CIs of overweight and grade 1-3 obesity by selected factors in men aged 40 years or older in NHANES 2001-2010.

\begin{tabular}{|c|c|c|c|c|c|}
\hline Characteristics & $\begin{array}{l}\text { Overweight } \\
(n=3,281)\end{array}$ & $\begin{array}{c}\text { Grade } 1 \\
\text { obesity }(n=1,729)\end{array}$ & $\begin{array}{c}\text { Grade } 2 \\
\text { obesity }(n=599)\end{array}$ & $\begin{array}{c}\text { Grade } 3 \\
\text { obesity }(n=275)\end{array}$ & $P$ value ${ }^{*}$ \\
\hline \multicolumn{6}{|l|}{ Age } \\
\hline Middle-aged & $43.1(41.0,45.2)$ & $22.8(21.2,24.5)$ & $8.4(7.3,9.5)$ & $4.1(3.4,4.9)$ & \multirow{2}{*}{0.038} \\
\hline Elderly & $42.6(40.8,44.5)$ & $22.8(21.2,24.4)$ & $7.7(6.6,8.8)$ & $2.9(2.1,3.6)$ & \\
\hline \multicolumn{6}{|l|}{ Race } \\
\hline Hispanic & $48.3(45.5,51.2)$ & $23.2(21.0,25.5)$ & $7.7(6.1,9.3)$ & $2.4(1.6,3.2)$ & \multirow{4}{*}{$<0.0001$} \\
\hline Non-Hispanic white & $43.6(41.7,45.5)$ & $23.1(21.5,24.7)$ & $8.4(7.3,9.5)$ & $3.7(3.0,4.3)$ & \\
\hline Non-Hispanic black & $35.6(33.2,38.0)$ & $22.1(19.6,24.6)$ & $8.7(7.1,10.2)$ & $5.4(4.2,6.5)$ & \\
\hline Other & $34.4(28.0,40.9)$ & $17.6(11.3,23.9)$ & $4.4(0.3,8.5)$ & $3.2(0.4,6.0)$ & \\
\hline \multicolumn{6}{|l|}{ Education } \\
\hline College or above & $43.8(41.5,46.0)$ & $22.8(21.3,24.4)$ & $8.3(7.0,9.6)$ & $3.3(2.5,4.1)$ & \multirow{2}{*}{0.398} \\
\hline High school or below & $41.8(39.5,44.1)$ & $22.8(21.0,24.6)$ & $8.0(6.7,9.3)$ & $4.2(3.4,5.1)$ & \\
\hline \multicolumn{6}{|l|}{ Poverty } \\
\hline No & $43.0(41.4,44.6)$ & $23.3(21.9,24.7)$ & $8.4(7.4,9.3)$ & $3.8(3.2,4.4)$ & \multirow{2}{*}{$<0.0001$} \\
\hline Yes & $39.6(35.8,43.5)$ & $19.0(15.7,22.2)$ & $7.6(5.3,9.9)$ & $2.6(1.2,4.0)$ & \\
\hline \multicolumn{6}{|l|}{ Alcohol use } \\
\hline No & $37.9(34.6,41.3)$ & $23.2(20.2,26.2)$ & $8.8(6.4,11.2)$ & $6.3(4.5,8.1)$ & \multirow{2}{*}{0.0005} \\
\hline Yes & $44.0(42.3,45.8)$ & $22.9(21.5,24.2)$ & $8.0(7.0,9.0)$ & $3.3(2.7,3.9)$ & \\
\hline \multicolumn{6}{|l|}{ Smoking } \\
\hline Nonsmoking & $44.2(41.8,46.6)$ & $21.4(19.2,23.7)$ & $9.2(7.5,10.9)$ & $4.4(3.5,5.3)$ & \multirow{3}{*}{$<0.0001$} \\
\hline Previous smoking & $45.8(43.2,48.3)$ & $24.6(22.6,26.6)$ & $8.4(6.8,10.0)$ & $3.3(2.4,4.2)$ & \\
\hline Current smoking & $38.0(35.0,41.0)$ & $22.5(19.9,25.0)$ & $6.8(5.6,8.1)$ & $3.5(2.4,4.5)$ & \\
\hline
\end{tabular}

Note: sampling weights were incorporated into data analyses.

${ }^{*} P$ value is for testing the significance of association of individual characteristics with grades of obesity.

NHANES: National Health and Examination Survey.

Standard errors were estimated with survey procedures using Taylor series linearization. Rao-Scott chi-square statistics were provided for testing the significance of associations of individual factors with grades of obesity and DPC, and a $P$ value of less than 0.05 was considered statistically significant. To examine the associations of grade 2 and 3 obesity with the risk of DPC, unadjusted odds ratios (OR), adjusted OR, and their 95\% CIs were derived by conducting weighted logistic regression models, controlled for the groups of confounding factors. Statistical analyses were performed using SAS software version 9.2 (SAS Institute Inc, Cary, North Carolina).

\section{Results}

Average age of selected men in this study was $56.08 \pm 0.19$ years; $65.52 \pm 0.72 \%$ of men were middle-aged and $34.48 \pm$ $0.72 \%$ were elderly. Hispanics accounted for $9.28 \pm 0.95 \%$ and non-Hispanic blacks accounted for $9.30 \pm 0.66 \%$. The rates of grade 2 and 3 obesity were $8.16 \pm 0.45 \%$ and $3.70 \pm 0.27 \%$, respectively. The overall rate of DPC in the study sample was $3.05 \pm 0.22 \%$.

Age, race, poverty, alcohol use, and smoking were significantly associated with grades of BMI (all $P<0.05$, Table 1 ), showing the confounding influence in the association of grades of BMI with the odds of DPC. Elderly men, poor men, and men with alcohol use and current smoking were more likely to have lower grade to normal weight. Among men grouped by selected factors, the overweight rate was highest in each group from $34.4 \%$ (95\% CI: $28.0-40.9 \%$ ) in men with other race to $48.3 \%$ (95\% CI: $45.5-51.2 \%$ ) in Hispanics. Overall, the rates of grades of BMI decreased significantly from overweight to grade 3 obesity except for the groups of men with other race and men without drinking alcohol.

Table 2 lists the unadjusted rates and 95\% CIs of DPC by selected factors in middle-aged and elderly men in NHANES 2001-2010. From this table, we can see that age, race, poverty status, alcohol use, and smoking status were significantly associated with the unadjusted rates of DPC (all $P<0.05$ ). Compared with middle-aged men, elderly men had higher rate of DPC (7.96\%, 95\% CI: $6.91-9.01 \%$ versus $0.46 \%, 95 \%$ CI: $0.15-0.78 \%)$. Non-Hispanic blacks had higher rate of DPC than Hispanics. The rates of DPC were lower in men who are poor, drinking alcohol, and currently smoking than men in the corresponding reference groups (1.57\% versus $3.13 \%$, $2.87 \%$ versus $4.20 \%$, and $1.18 \%$ versus $2.79 \%$, resp.).

The unadjusted rates of DPC over grades of BMI are presented in Figure 1. Men with grade 2 and 3 obesity appeared to have lower rates of DPC, but the overall difference in rates 
TABLE 2: Unadjusted rates and 95\% CIs of diagnosed prostate cancer by selected factors in men aged 40 years or older in NHANES 20012010.

\begin{tabular}{lcc}
\hline Characteristics & $\begin{array}{c}\text { Diagnosed prostate } \\
\text { cancer }(\%)\end{array}$ & $P$ value* \\
\hline Age & $0.46(0.15,0.78)$ & $<0.0001$ \\
$\quad$ Middle-aged & $7.96(6.91,9.01)$ & \\
$\quad$ Elderly & & \\
Race & $1.37(0.78,1.97)$ & \\
$\quad$ Hispanic & $3.22(2.69,3.75)$ & 0.0001 \\
$\quad$ Non-Hispanic white & $3.85(3.10,4.61)$ & \\
$\quad$ Non-Hispanic black & $1.78(0.53,3.04)$ & \\
$\quad$ Other & & \\
Education & $2.97(2.26,3.69)$ & \\
College or above & $3.12(2.68,3.57)$ & \\
High school or below & & \\
Poverty & $3.13(2.64,3.62)$ & \\
$\quad$ No & $1.57(0.97,2.18)$ & \\
Yes & & 0.0003 \\
Alcohol use & $4.20(2.95,5.45)$ & \\
No & $2.87(2.38,3.37)$ & \\
Yes & & \\
Smoking & $2.79(2.19,3.39)$ & \\
Nonsmoking & $4.97(4.09,5.86)$ & \\
Previous smoking & $1.18(0.65,1.71)$ & \\
Current smoking & & \\
\hline
\end{tabular}

Note: sampling weights were incorporated into data analyses.

${ }^{*} P$ value is for testing the significance of association of individual characteristics with the risk of prostate cancer.

NHANES: National Health and Nutrition Examination Survey.

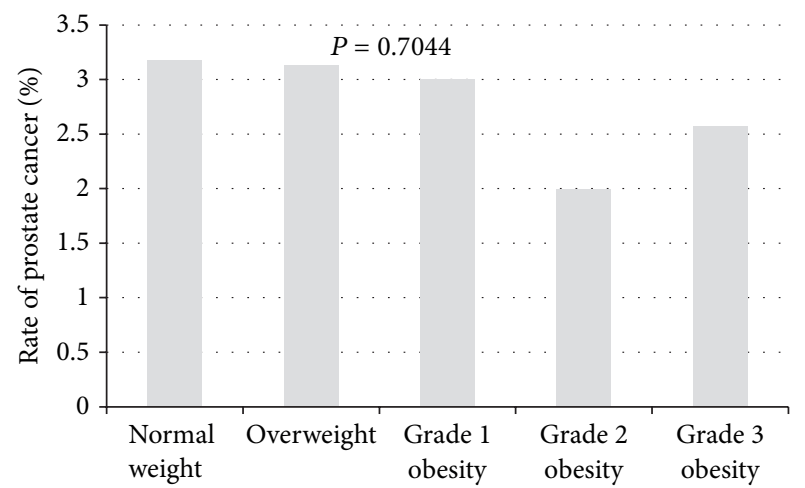

FIGURE 1: Rates of diagnosed prostate cancer over grades of body mass index.

of DPC did not attain the significance level compared with men with normal weight $(P=0.7044)$.

Odds ratios and 95\% CIs of DPC for grade 2 and grade 3 obesity calculated from weighted logistic regression models are shown in Table 3 . This table shows that grade 2 and 3 obesity were not significantly associated with the odds of DPC after adjustment for different groups of potential confounding factors (Model I-Model V). Furthermore, there were no associations between morbid obesity (grade 2 and 3 obesity combined) or general obesity (grade 1-3 obesity combined) and the odds of DPC. In the complete model (Model V), non-Hispanic black men were more likely and Hispanic men were less likely to have DPC than nonHispanic white men. Poverty status and alcohol use were not associated with the odds of DPC, but current smoking was still associated with lower odds of DPC after adjustment for other factors in the model (results not shown).

\section{Discussion}

In the present study, we examined the association among grade 2 obesity, grade 3 obesity, and the odds of DPC in men aged 40 years or older using the data from NHANES 20012010. The results showed that whether or not we adjusted for the confounding influence from age, gender, race/ethnicity, alcohol use, or smoking status, neither grade 2 nor grade 3 obesity was significantly associated with the odds of DPC in the middle-aged and elderly men. This indicated that morbid obesity (BMI $\geq 35 \mathrm{~kg} / \mathrm{m}^{2}$ ) did not appear to be linked to DPC. We combined different grades of obesity into general obesity (BMI $\left.\geq 30 \mathrm{~kg} / \mathrm{m}^{2}\right)$ and then examined the association between general obesity and DPC. Like morbid obesity, general obesity was not found to be associated with the odds of DPC in this study.

Many studies have shown that obesity is not associated with the prostate cancer risk [12-14]. Whether grade 2 and grade 3 obesity were associated with the odds of prostate cancer has not been investigated. As far as we know, our study is the first study that has investigated the associations of grade 2 and 3 obesity with the odds of DPC in middleaged and elderly men. We anticipated that grades of obesity might differentially affect the odds of prostate cancer, and grade 2 and 3 obesity had significant association with the odds of total DPC. However, in the present study, we did not find that grade 2 and grade 3 obesity were associated with the odds of total DPC. NHANES does not provide information on the grades or stages of prostate cancer. A study with the disease grade or stage information is needed to further investigate whether grade 2 and grade 3 obesity are associated with the grades/stages of DPC. It is likely that morbid obesity is associated with the increased risk of high-graded disease and the reduced risk of low-grade disease while it is not related to the risk of the disease.

The result of no association between obesity and DPC in this study is consistent with the findings from several previous studies [12-14], and nonsignificant association between morbid obesity and DPC (grade 2 and 3 obesity) further supports the previous findings in the sense that not only general obesity but also grade 2 and grade 3 obesity were not associated with the odds of DPC. Although some studies have reported associations of obesity with a lower risk of total cancer [19] or a higher risk of high-grade cancer [20], our study, along with other studies, has found no association between obesity/morbid obesity and total prostate cancer [12-14]. Conclusive evidence has shown that obesity is associated 
TABLE 3: Adjusted odds ratios and 95\% CIs of diagnosed prostate cancer associated with grades of obesity in men aged 40 years or older in NHANES 2001-2010.

\begin{tabular}{|c|c|c|c|c|c|}
\hline \multirow{2}{*}{ Levels of obesity } & \multicolumn{5}{|c|}{ Odds ratios and $95 \%$ CIs } \\
\hline & Model I & Model II & Model III & Model IV & Model V \\
\hline $\begin{array}{l}\text { Overweight } \\
\left(25 \leq \mathrm{BMI}<30 \mathrm{~kg} / \mathrm{m}^{2}\right)\end{array}$ & $0.99(0.69,1.42)$ & $1.05(0.74,1.50)$ & $1.05(0.74,1.50)$ & $1.04(0.72,1.52)$ & $0.98(0.66,1.44)$ \\
\hline $\begin{array}{l}\text { Grade } 1 \text { obesity } \\
\left(30 \leq \mathrm{BMI}<35 \mathrm{~kg} / \mathrm{m}^{2}\right)\end{array}$ & $0.95(0.66,1.37)$ & $1.00(0.70,1.42)$ & $1.00(0.70,1.43)$ & $0.93(0.64,1.36)$ & $0.89(0.60,1.32)$ \\
\hline $\begin{array}{l}\text { Grade } 2 \text { obesity } \\
\left(35 \leq \mathrm{BMI}<40 \mathrm{~kg} / \mathrm{m}^{2}\right)\end{array}$ & $0.62(0.36,1.07)$ & $0.67(0.38,1.17)$ & $0.67(0.38,1.18)$ & $0.69(0.39,1.24)$ & $0.63(0.35,1.14)$ \\
\hline $\begin{array}{l}\text { Grade } 3 \text { obesity } \\
\left(\mathrm{BMI} \geq 40 \mathrm{~kg} / \mathrm{m}^{2}\right)\end{array}$ & $0.81(0.28,2.30)$ & $1.03(0.35,3.03)$ & $1.04(0.36,3.05)$ & $1.09(0.37,3.20)$ & $1.08(0.36,3.18)$ \\
\hline $\begin{array}{l}\text { Morbid obesity } \\
\left(\mathrm{BMI} \geq 35 \mathrm{~kg} / \mathrm{m}^{2}\right)\end{array}$ & $0.68(0.39,1.17)$ & $0.77(0.44,1.34)$ & $0.77(0.44,1.35)$ & $0.80(0.45,1.41)$ & $0.75(0.42,1.33)$ \\
\hline $\begin{array}{l}\text { General obesity } \\
\left(\mathrm{BMI} \geq 30 \mathrm{~kg} / \mathrm{m}^{2}\right)\end{array}$ & $0.86(0.62,1.18)$ & $0.92(0.67,1.27)$ & $0.93(0.67,1.28)$ & $0.89(0.63,1.25)$ & $0.84(0.60,1.19)$ \\
\hline
\end{tabular}

Note: reference category in the first column is normal weight $\left(\mathrm{BMI}<25 \mathrm{~kg} / \mathrm{m}^{2}\right)$.

Model I: unadjusted model including the primary factor (obesity status) only; Model II: Model I plus age and race; Model III: Model II plus education; Model IV: Model III plus poverty status; Model V: Model IV plus alcohol use and smoking use; NHANES: National Health and Nutrition Examination Survey; CI: confidence interval; BMI: body mass index.

with an increased risk of prostate cancer mortality [21, 22]. One potential explanation for these conflicting results is that obesity may differentially affect the development of aggressive and nonaggressive prostate cancers [23]. Of the studies that stratified by grade, there is growing evidence that obesity may increase the risk of high-grade disease and decrease the risk of low-grade disease $[11,20]$. The study of obesity that does not stratify by cancer grade may detect little or no association of obesity with this cancer's risk. Nevertheless, the differences in study populations, definitions of obesity, and classifications of aggressive disease may make summary interpretation of this literature difficult.

One of several mechanisms that could explain the associations of obesity/morbid obesity with prostate cancer risk is through steroid hormones. In men, obesity is associated with various endocrine aberrations and grade 2 and grade 3 obesity are associated with more severe aberration, including substantially higher serum estrogen [24] and substantially lower levels of testosterone and sex hormone-binding globulin [25]. In contrast to the accepted dogma that high testosterone levels increase the risk, more recent studies of steroid hormones and prostate cancer risk have shown that high levels of testosterone are associated with reduced risk of high-grade and increased risk of low-grade disease [26], and high levels of estradiol are associated with decreased risk of "nonaggressive" cancer but not aggressive cancer [27]. Thus, obesity-related changes in the overall pattern of sex hormone concentrations may support the biological plausibility of our finding that grade 2 or grade 3 obesity was not associated with the odds of the total DPC with high grade and low grade of cancer combined.

Current smoking has been inversely associated with the odds of DPC in our study, which conflicts with the reports from the previous studies $[28,29]$. The possible explanation is that NHANES is a program with a cross-sectional design. The men who were smoking when being diagnosed as having prostate cancer might realize the risk of smoking related to prostate cancer and quit smoking after that. Hence the inverse association between smoking and the risk of prostate cancer in this study may not reflect the association of current smoking with DPC but the association of DPC with current smoking status. While it has never been directly shown that smoking causes prostate cancer, previous studies have shown that smoking is associated with higher rates of prostate cancer [28]. In addition, studies have shown that men who smoke have a greater risk of dying of their prostate cancer [29].

Continuous NHANES survey was a large stratified, multistage national survey of noninstitutionalized participants with complex probability design. By considering the sampling weights, our results could be directly generalized to the general population of middle-aged and elderly men in the United States. There were several limitations in this study. First, NHANES was a cross-sectional survey without any follow-up information of participants. While the findings in the study could reflect the association of grade 2 or grade 3 obesity with the odds of DPC, they could not be used for causal effects of grade 2 and 3 obesity on the risk of DPC in the population of men with follow-ups in the cohort design. Second, there might be unrecognized measurements confounding the association between grade 2 and 3 obesity and prostate cancer odds although we had adjusted for the potential confounding influence of age, gender, education, alcohol use, and smoking status. Finally, there was no detailed grade/stage information for prostate cancer in the NHANES survey. This prevented us from examining the association between grade 2 and 3 obesity and the odds of high-grade and low-grade prostate cancer.

In conclusion, there were no significant associations between grade 2 or grade 3 obesity and the odds of DPC in this study. Compared with men with normal weight, obese or morbidly obese men with similar odds of prostate cancer might not increase the sensitivity of disease screening, 
leading to delayed diagnosis of aggressive types of disease as accumulative evidence has shown that obese men are associated with an increased risk of high-grade and thus clinically significant prostate cancer $[11,20]$. As the prevalence of obesity in the US is high and the rates of obesity around the world are increasing, the population-attributable risk for high-grade prostate cancer due to obesity will be substantial. Reduced risk of prostate cancer should be added to the list of benefits of weight control, and this message may motivate men, especially men with a family history of prostate cancer, to better follow recommendations for healthful diets and increased physical activity. In addition, the significant association between obesity and risk of high-grade prostate cancer may indicate that grade 2 or grade 3 obesity is linked to substantially higher risk of high-grade disease. Whether they are actually associated with high-grade or low-grade prostate cancer will need to be further investigated.

\section{Conflict of Interests}

The authors declare no conflict of interests.

\section{References}

[1] A. W. Hsing, L. C. Sakoda, and S. C. Chua Jr., "Obesity, metabolic syndrome, and prostate cancer," The American Journal of Clinical Nutrition, vol. 86, supplement, pp. 843S-857S, 2007.

[2] A. Jemal, R. Siegel, E. Ward, T. Murray, J. Xu, and M. J. Thun, "Cancer statistics, 2007," CA: A Cancer Journal for Clinicians, vol. 57, no. 1, pp. 43-66, 2007.

[3] Prostate Cancer Overview, American Cancer Society, 2013, http://www.cancer.org/cancer/prostatecancer/overviewguide/ prostate-cancer-overview-key-statistics.

[4] D. G. Bostwick, H. B. Burke, D. Djakiew et al., "Human prostate cancer risk factors," Cancer, vol. 101, no. 10, supplement, pp. 2371-2490, 2004.

[5] A. W. Hsing and A. P. Chokkalingam, "Prostate cancer epidemiology," Frontiers in Bioscience, vol. 11, no. 2, pp. 1388-1413, 2006.

[6] S. K. Park, L. C. Sakoda, D. Kang et al., "Rising prostate cancer rates in South Korea," Prostate, vol. 66, no. 12, pp. 1285-1291, 2006.

[7] E. A. Finkelstein, J. G. Trogdon, J. W. Cohen, and W. Dietz, "Annual medical spending attributable to obesity: payer-and service-specific estimates," Health Affairs, vol. 28, no. 5, pp. w822-w831, 2009.

[8] National Institutes of Health, "Clinical guidelines on the identification, evaluation, and treatment of overweight and obesity in adults: the evidence report," Obesity Research, vol. 6, supplement 2, pp. 51S-209S, 1998.

[9] B. D. Bradbury, J. B. Wilk, and J. A. Kaye, "Obesity and the risk of prostate cancer (United States)," Cancer Causes and Control, vol. 16, no. 6, pp. 637-641, 2005.

[10] M. P. Porter and J. L. Stanford, "Obesity and the risk of prostate cancer," Prostate, vol. 62, no. 4, pp. 316-321, 2005.

[11] Z. Gong, M. L. Neuhouser, P. J. Goodman et al., "Obesity, diabetes, and risk of prostate cancer: results from the prostate cancer prevention trial," Cancer Epidemiology Biomarkers and Prevention, vol. 15, no. 10, pp. 1977-1983, 2006.
[12] I.-M. Lee, H. D. Sesso, and R. S. Paffenbarger Jr., "A prospective cohort study of physical activity and body size in relation to prostate cancer risk (United States)," Cancer Causes and Control, vol. 12, no. 2, pp. 187-193, 2001.

[13] F. Jonssoni, A. Wolk, N. L. Pedersen et al., "Obesity and hormone-dependent tumors: cohort and co-twin control studies based on the Swedish Twin Registry," International Journal of Cancer, vol. 106, no. 4, pp. 594-599, 2003.

[14] C. Rodriguez, A. V. Patel, E. E. Calle, E. J. Jacobs, A. Chao, and M. J. Thun, "Body mass index, height, and prostate cancer mortality in two large cohorts of adult men in the United States," Cancer Epidemiology Biomarkers and Prevention, vol. 10, no. 4, pp. 345-353, 2001.

[15] A. Engeland, S. Tretli, and T. Bjørge, "Height, body mass index, and prostate cancer: a follow-up of 950000 Norwegian men," British Journal of Cancer, vol. 89, no. 7, pp. 1237-1242, 2003.

[16] Centers for Disease Control and Prevention (CDC). National Center for Health Statistics (NCHS), National Health and Nutrition Examination Survey Data, U.S. Department of Health and Human Services, Centers for Disease Control and Prevention, Hyattsville, Md, USA, 1999-2010, http://www.cdc.gov/ nchs/nhanes.htm.

[17] Centers for Disease Control and Prevention (CDC). National Center for Health Statistics (NCHS), National Health and Nutrition Examination Survey Data, U.S. Department of Health and Human Services, Centers for Disease Control and Prevention, Hyattsville, Md, USA, 2013, http://www.cdc.gov/nchs/ tutorials/nhanes/SurveyDesign/SampleDesign/intro.htm.

[18] WHO Expert Committee on Physical Status, Physical Status: The Use and Interpretation of Anthropometry, World Health Organization, Geneva, Switzerland, 1995.

[19] E. Giovannucci, E. B. Rimm, Y. Liu et al., "Body mass index and risk of prostate cancer in U.S. health professionals," Journal of the National Cancer Institute, vol. 95, no. 16, pp. 1240-1244, 2003.

[20] R. J. MacInnis, D. R. English, D. M. Gertig, J. L. Hopper, and G. G. Giles, "Body size and composition and prostate cancer risk," Cancer Epidemiology Biomarkers and Prevention, vol. 12, no. 12, pp. 1417-1421, 2003.

[21] C. Rodriguez, A. V. Patel, E. E. Calle, E. J. Jacobs, A. Chao, and M. J. Thun, "Body mass index, height, and prostate cancer mortality in two large cohorts of adult men in the United States," Cancer Epidemiology Biomarkers and Prevention, vol. 10, no. 4, pp. 345-353, 2001.

[22] E. E. Calle, C. Rodriguez, K. Walker-Thurmond, and M. J. Thun, "Overweight, obesity, and mortality from cancer in a prospectively studied cohort of U.S. adults," The New England Journal of Medicine, vol. 348, no. 17, pp. 1625-1638, 2003.

[23] S. J. Freedland, E. Giovannucci, and E. A. Platz, "Are findings from studies of obesity and prostate cancer really in conflict?" Cancer Causes and Control, vol. 17, no. 1, pp. 5-9, 2006.

[24] E. E. Calle and R. Kaaks, "Overweight, obesity and cancer: epidemiological evidence and proposed mechanisms," Nature Reviews Cancer, vol. 4, no. 8, pp. 579-591, 2004.

[25] R. Pasquali, F. Casimirri, S. Cantobelli et al., "Effect of obesity and body fat distribution on sex hormones and insulin in men," Metabolism, vol. 40, no. 1, pp. 101-104, 1991.

[26] G. Severi, H. A. Morris, R. J. MacInnis et al., "Circulating steroid hormones and the risk of prostate cancer," Cancer Epidemiology Biomarkers and Prevention, vol. 15, no. 1, pp. 86-91, 2006.

[27] C. Chen, N. S. Weiss, F. Z. Stanczyk et al., "Endogenous sex hormones and prostate cancer risk: a case-control study 
nested within the carotene and retinol efficacy trial," Cancer Epidemiology Biomarkers and Prevention, vol. 12, no. 12, pp. 1410-1416, 2003.

[28] L. A. Plaskon, D. F. Penson, T. L. Vaughan, and J. L. Stanfordz, "Cigarette smoking and risk of prostate cancer in middle-aged men," Cancer Epidemiology Biomarkers and Prevention, vol. 12, no. 7, pp. 604-609, 2003.

[29] S. A. Kenfield, M. J. Stampfer, J. M. Chan, and E. Giovannucci, "Smoking and prostate cancer survival and recurrence," The Journal of the American Medical Association, vol. 305, no. 24, pp. 2548-2555, 2011. 


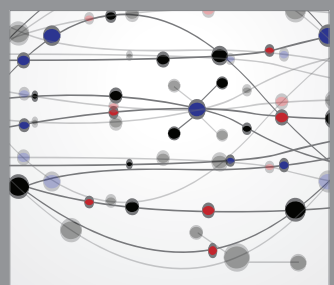

The Scientific World Journal
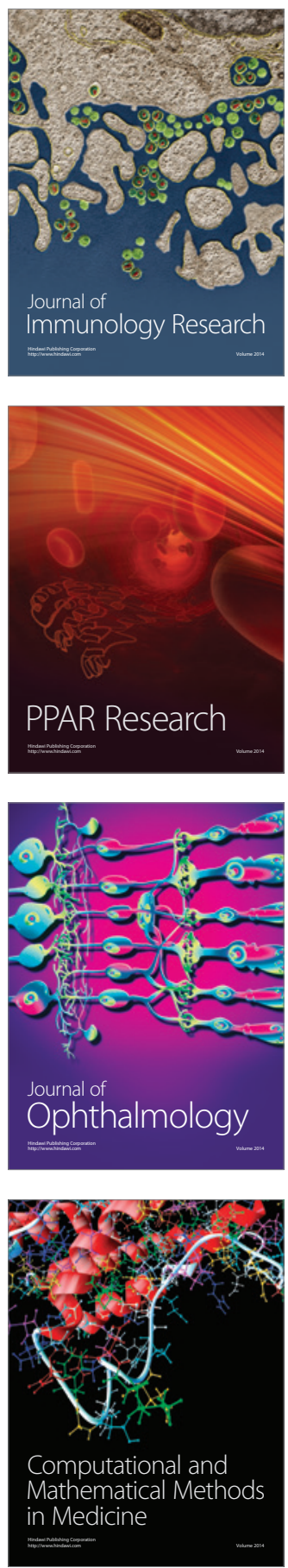

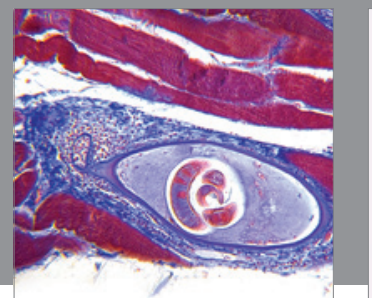

Gastroenterology

Research and Practice
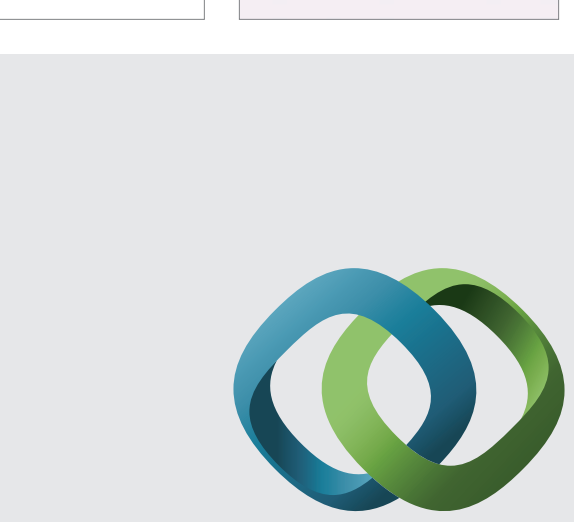

\section{Hindawi}

Submit your manuscripts at

http://www.hindawi.com
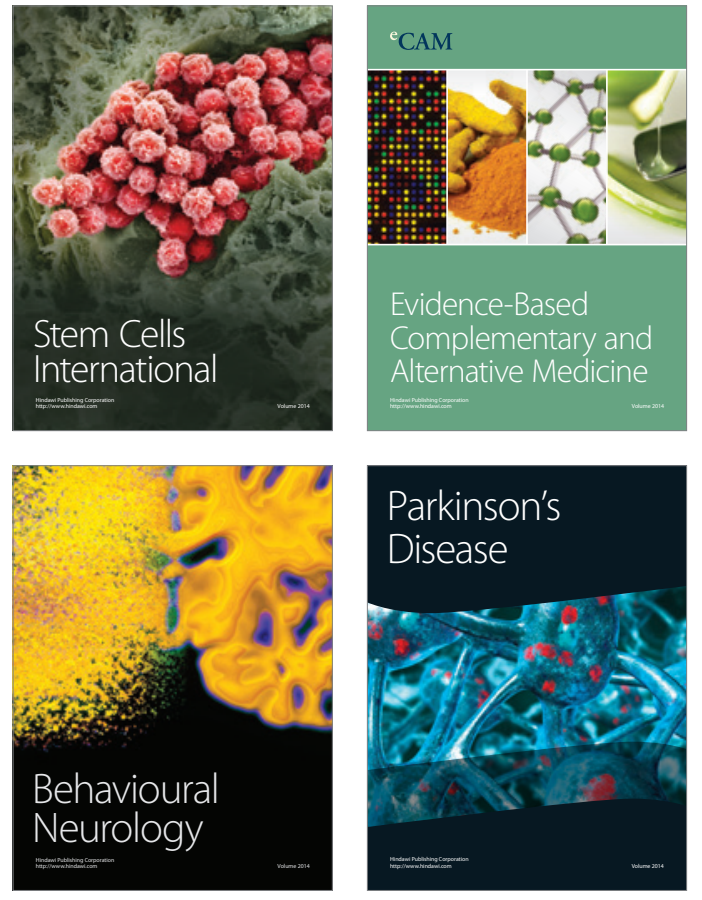
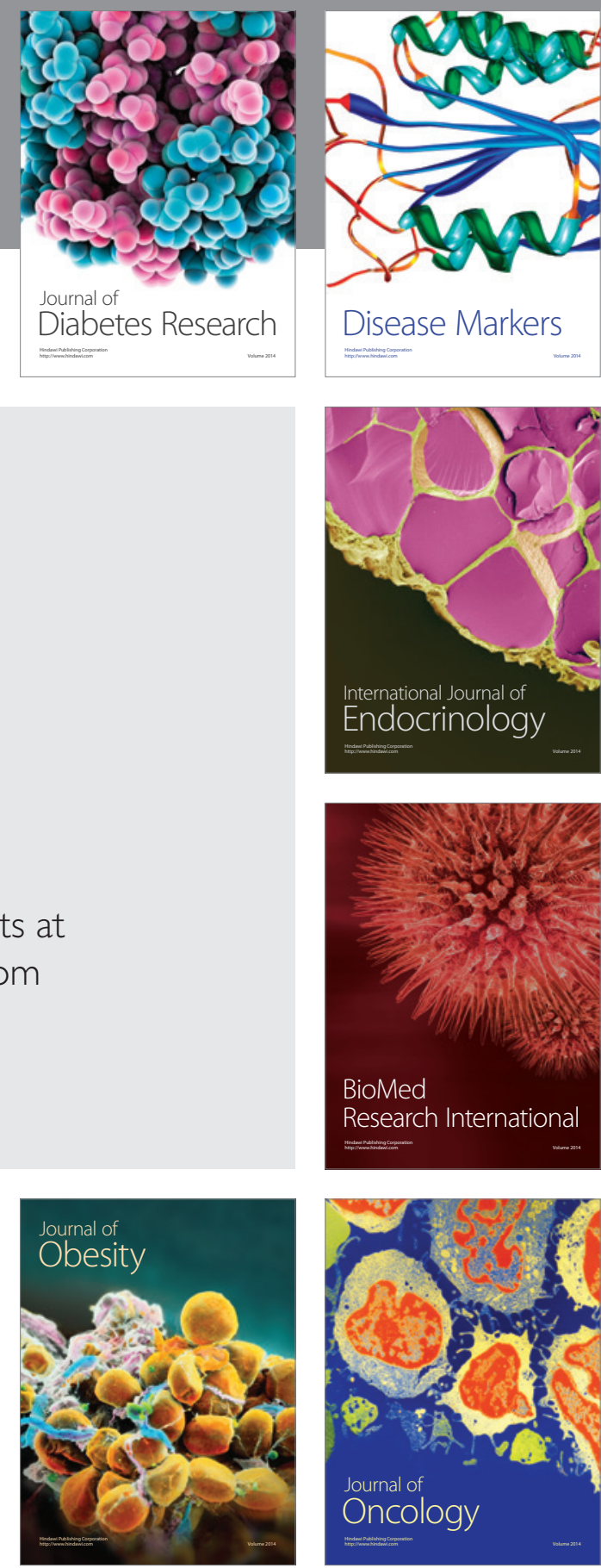

Disease Markers
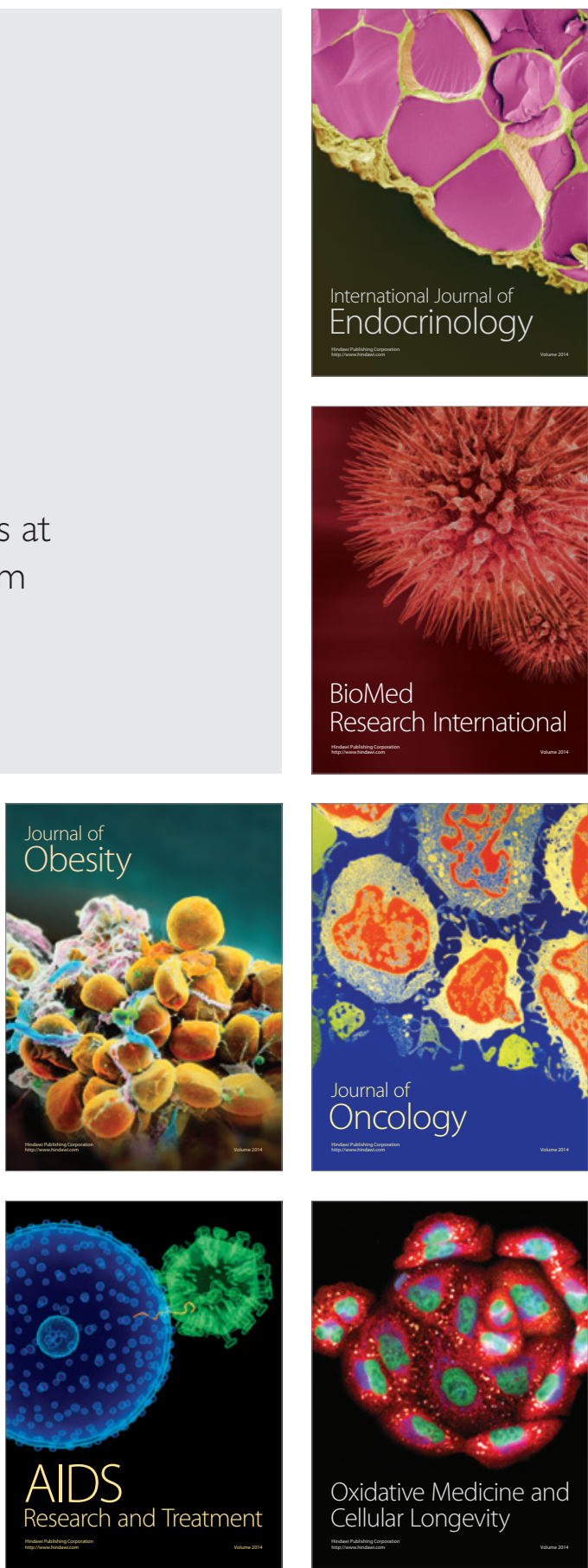